\title{
Private Pilot Incidents by Pilot Age and Recentness of Medical Certification
}

\author{
Donald R. Morris
}

Southern Illinois University

\begin{abstract}
The link between recentness of medical certification and increased likelihood of involvement in an NTSB incident has been previously reported (Morris, 2016). This study investigated this phenomena across pilot ages. Age groups were fit to an exponential-linear decay model using the least squares method of best fit. Standard errors were reported in order to verify quality of fit. Individual best fit lines were graphed and shown for demographic groups. Best fit lines from across groups were then compared.

This study verified the relationship between recent third class medical certification and increased likelihood of being involved in incidents for all age demographics. The study also showed that younger pilots have a marked initial increase in risk when compared to their older counterparts. It also showed that as pilots age, the relationship between recent medical certification and increased risk was significantly reduced in strength but still was very present.
\end{abstract}

\section{Introduction}

Statisticians have long cautioned that correlation does not imply causation. There are few better apparent examples of this fact than the correlation between how recently a private pilot has earned his or her third class medical and the likelihood of them being involved in an NTSB database incident. This relationship was recently reported in the Collegiate Aviation Review (Morris, 2016). The goal of this study is to analyze this data curve - shown in figure 1 - and to see how it varies depending on the age demographics of pilots.

Three possible reasons for this relationship were suggested in the original study. These included complacency due to recent medical certification, lack of recent flying experience for many of those with recent medical certification, and inconsistencies in the number of flight hours across time that are flown by private pilots (Morris, 2016).

It is important to note that the referenced study limited itself to private pilots with $3^{\text {rd }}$ class medicals. As such, this data represents a group of pilots that tend to fly when they want to fly. Therefore, it is believed that the number of hours they fly is very inconsistent compared to commercial pilots whose flight hours are flown as part of their employment. In support of this belief, Hunter's 1995 survey of pilot hours flown indicates that the standard deviation of numbers of hours flown in the last year by private pilots exceeds the average number of hours flown by private pilots - indicating highly inconsistent flying habits. Airline transport pilots in the same study did not demonstrate this same tendency. 


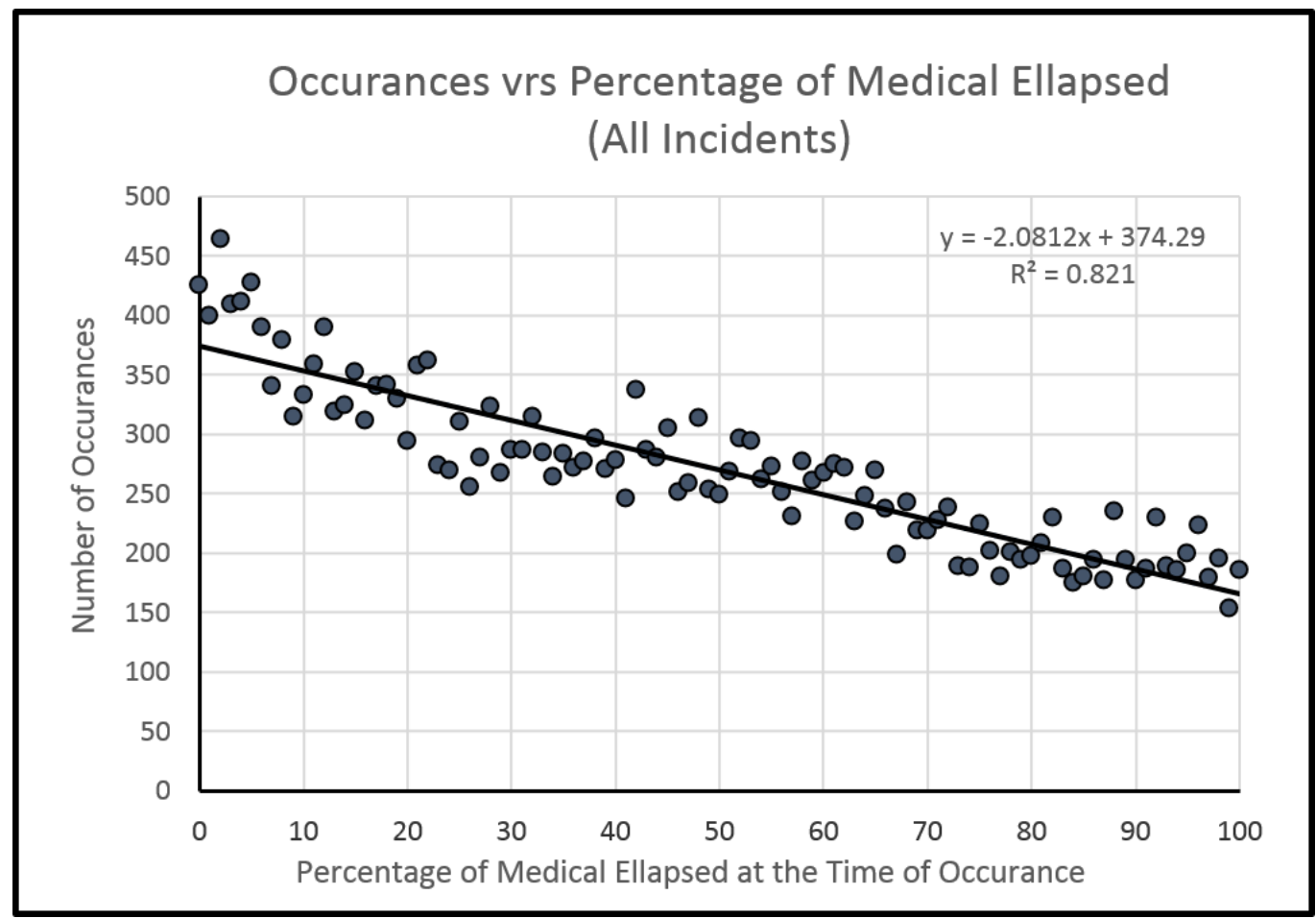

Figure 1: Likelihood of Being Involved in an Incident is Strongly Predicted by Recentness of Medical Certification (Image Source: Morris, 2016)

\section{Rationale}

Very little hard data is publicly available about the number of hours flown by the 'average' private pilot (Ohare \& Chalmers, 1999). Unless a pilot earns additional ratings or certificates, they may never report how many hours they have flown. Even if they do progress through the system, the hourly totals collected on form 8710-1 under the Record of Pilot Time contain no time-based information. Except in rare cases, this provides only limited information regarding flight times for pilots.

In the US, there is no mechanism for collection of private pilot flight time data. However, part 830 of the NTSB regulations require a mandatory accident and incident reporting. These mandatory reports are the basis for the data analyzed in this study. Although the exact ratios and mechanisms of the link are a subject of intensive study (Knecht, 2015; Ison, 2015; Ohare \& Chalmers, 1999), there is an indisputable correlation between the incidents recorded in the database and the number of hours flown by private pilots. A pilot that flies zero hours has a zero percent probability of being involved in an incident. It is the hope of the author that future studies will be able to combine the research validating this link with the research presented in this study to provide highly accurate average flight hour characteristic data for private pilots. As such, the emphasis of this study is on describing comparative trends across large amounts of data. 


\section{Data}

Because this study is a follow up of the previously mentioned study, the data used in this study is the same as the data for the previous study. Specifically, the dataset consists of all incidents and accidents in the NTSB database between January $1^{\text {st }}, 1982$ and April 30 ${ }^{\text {th }}, 2015$ in which the pilot held a valid third class medical at the time of the accident or incident. Some 26,987 distinct incidents or accidents are included in this dataset. Percentages of elapsed time on the most recent medical at the time of the incident were calculated based on the age of the pilot and the regulations at the time of the incident or accident. These regulations changed twice during the sample represented, increasing the duration of $3^{\text {rd }}$ class medicals for those under the age of 40 from 24 to 36 calendar months in 1996 and then to 60 calendar months in 2008.

The original curve was created by constructing histograms of incidents vs time. In this study, the data was further filtered into eight distinct age-based demographic categories. These included those under 20 years of age, those in their 20's, 30's, 40's, 50's, 60's, or 70's, and those beyond 79 years of age at the time of the incident or accident.

\section{Analysis}

As in the previous study, the $0 \%$ and $100 \%$ histogram buckets of each sample were also doubled in size to compensate for their effective one-half percentage point width as compared to the other buckets full percentage point width. The resulting sample sizes are noted in figure 2, along with a graphical depiction of their relative size. This sample very closely approximates a theoretical bell curve distribution. Note that adding the size of each bucket gives a total of 27293 - some 306 higher than the overall sample size. This discrepancy is directly related to the doubling of bucket sizes just mentioned.

In order to adjust for different sample sizes and to be able to compare data metrics directly, the populations were next scaled to the same number of individuals. Each of these samples was then graphed, and best-fit lines were calculated. A visual examination of the graphs revealed a transient exponential decrease in the curve, fading into a linear fit. The general trend showed a much stronger exponential component for younger pilots, fading out as pilots increased in age. This model was seen as being consistent with the proposed reasons for the curve. The exponential portion of the curve fit very well with the theoretical effects of increased complacency and lack of experience - both of which would be expected to diminish exponentially with time. The linear portion, then, would seem to relate to the uneven number of hours that private pilots fly across time. This theoretical curve is not very different than the log-linear model presented by Bazargan \& Guzhva in their 2007 study (as cited in Knecht, 2015). 


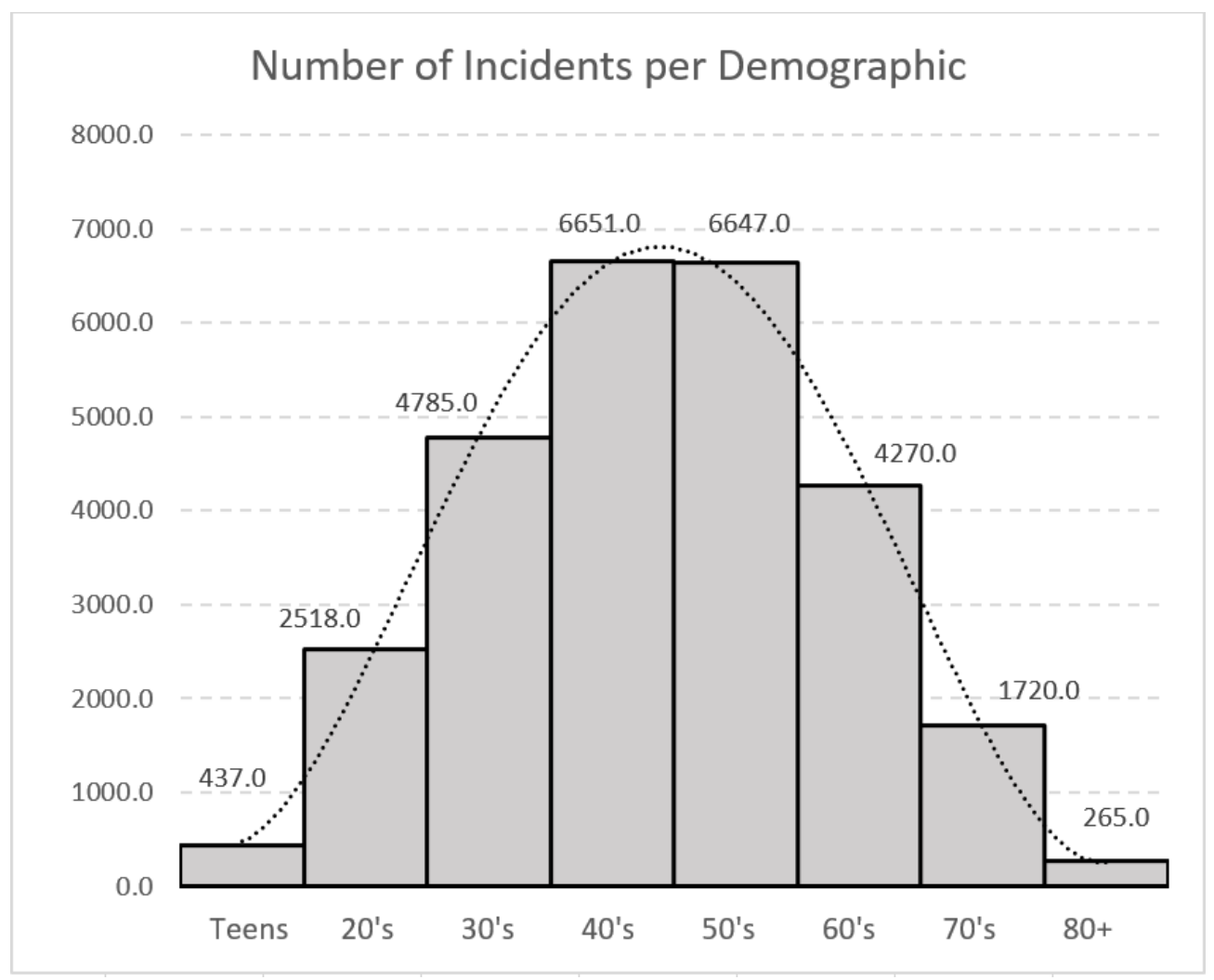

Figure 2: Graphical Depiction of Incidents Broken into Demographic Groups

Having decided on a theoretical curve based on the observed data, a custom best fit was calculated based upon the combined linear and exponential formula

$$
y=m x+b+A e^{-B x}
$$

where $m$ and $b$ are the familiar slope and intercept of the line, $A$ is the scale of the exponential component, and $B$ is the rate of exponential decay. Microsoft's Solver add-in pack for Excel was used to calculate this custom best fit line for each age group using the least squares method. Since the rate of decay was fairly consistent across the data, the entire set of data was analyzed as a block to determine which value of $\mathrm{B}$ would return the lowest standard error when used consistently across demographic groups. This value was then incorporated into the graphs. 

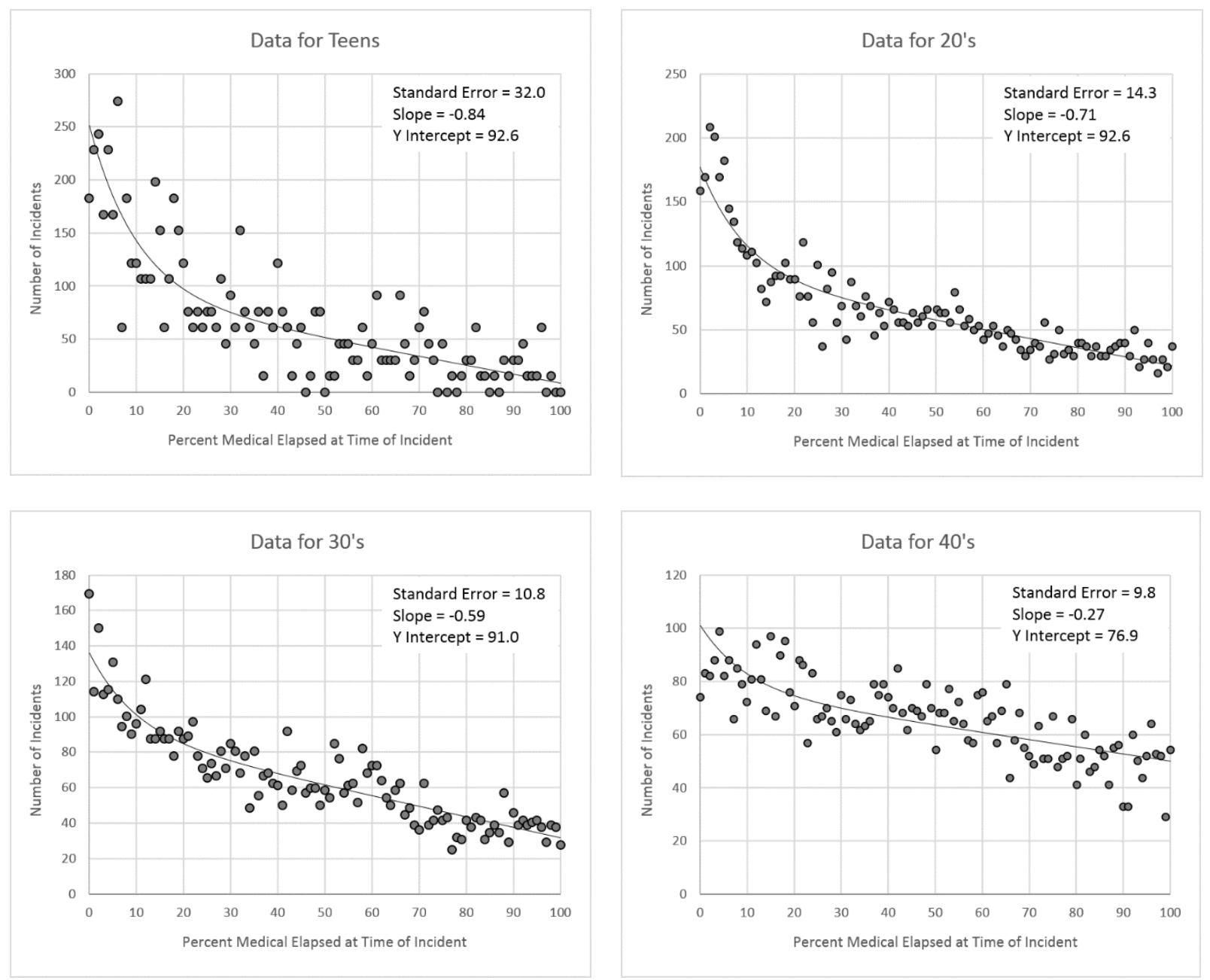

Figures 3-6: Data for Teens through 40's Showing Calculated Best Fits and Data

Finally, the scale of the exponential portion of the data fit - represented by $\mathrm{A}$ in the equation - was examined. As has been already noted, an observable trend existed where younger age groups had stronger exponential portions. Only one demographic group did not fit directly into this trend, and this was the 40-49 year olds. The strength of the apparent trend was judged to be sufficient to merit a best fit of best fits, and a variety of fits were tried. In the end, an exponential decay of $A$ compared to sample age provided the minimum standard error. Figures 3-10 show all the data graphs with trend line statistics. Figure 11 shows all the best fit lines plotted on a single graph. Note that its appearance is substantially different from the initial graph reported in the previous study. This is due to the fact that each demographic has now been weighted equally, and this strongly increased the number of incidents corresponding to the younger groups. This has significantly increased the size of the exponential component seen in the upper left of the graph. 

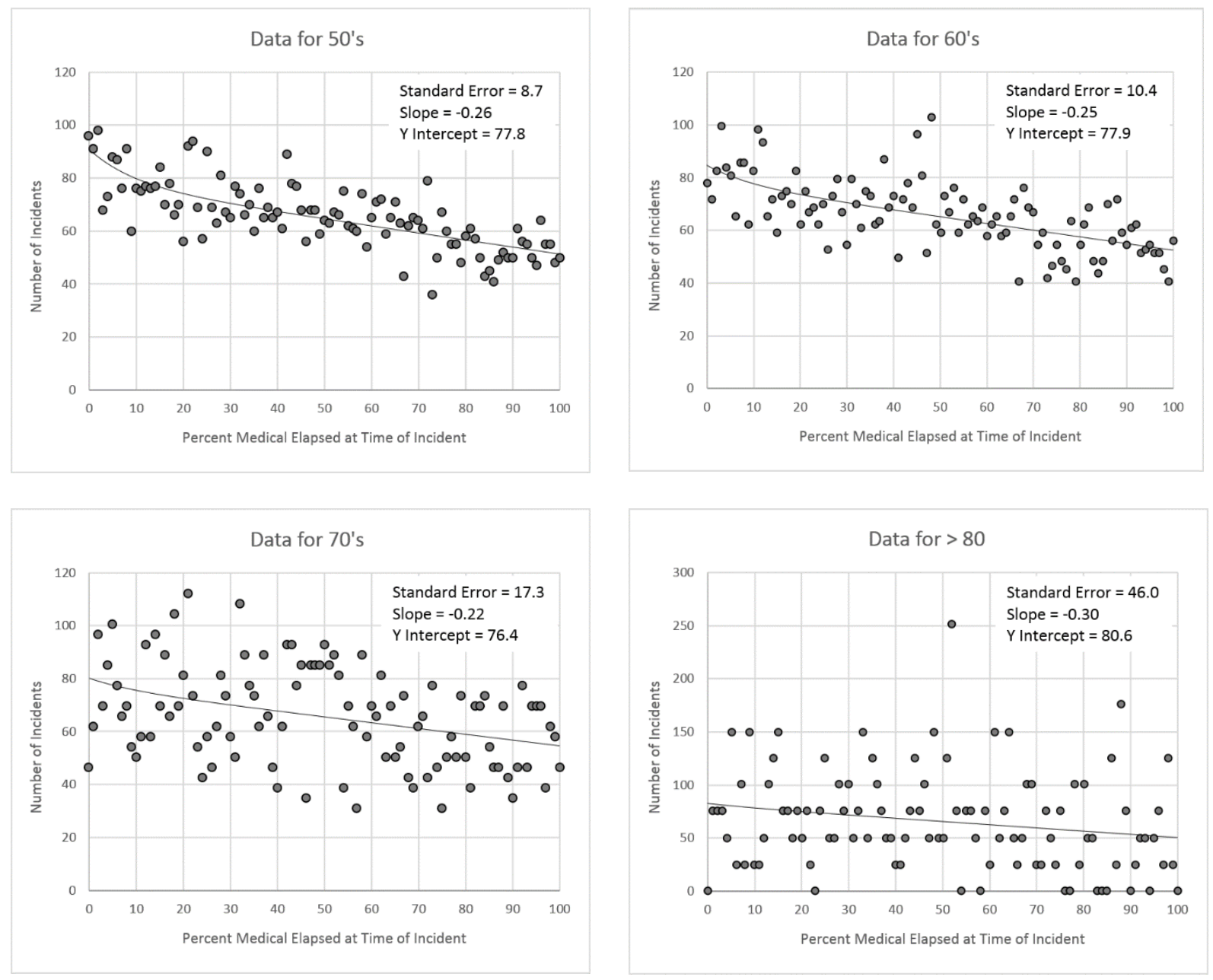

\section{Figures 7-10: Data for 50's through 80+ Showing Calculated Best Fits and Data}

In the previous article, $\mathrm{R}^{2}$ was used to analyze the best fit. In linear fits, $\mathrm{R}^{2}$ corresponds to the percentage of the data fit that can be directly described with the model. Unfortunately, $\mathrm{R}^{2}$ is a relatively meaningless statistic on non-linear fits such as were used in this study. Therefore, the standard error (S) was calculated instead. This standard error corresponds to the average number of points that each data point is away from the curve, and can be visualized as the standard deviation of each data point from the best fit curve. Lower values, therefore, indicate a better fit.

\section{Conclusions}

The most obvious conclusion that can be drawn from the data is that younger pilots show a much higher initial probability of being involved in an incident than their older counterparts. This is most likely explained by the fact that the younger pilot demographic groups contain far higher numbers of new pilots. Consider, for example, the teenage pilots. Most of them have only had one medical. In this demographic group, recent medical certification is almost 100\% associated with extremely low hours of flight time. As average age increases, so does the number of experienced pilots included in the sample (Ison, 2015). This means that recent medical certification in older demographic brackets is less strongly tied to very low flight hours of experience. This understanding correlates well 
to the data and provides a working explanation for its exponential decrease. Numerically, this decrease in initial incidents across demographics can be approximated by the expression $0.10 e^{-0.063 \times A g e}$.

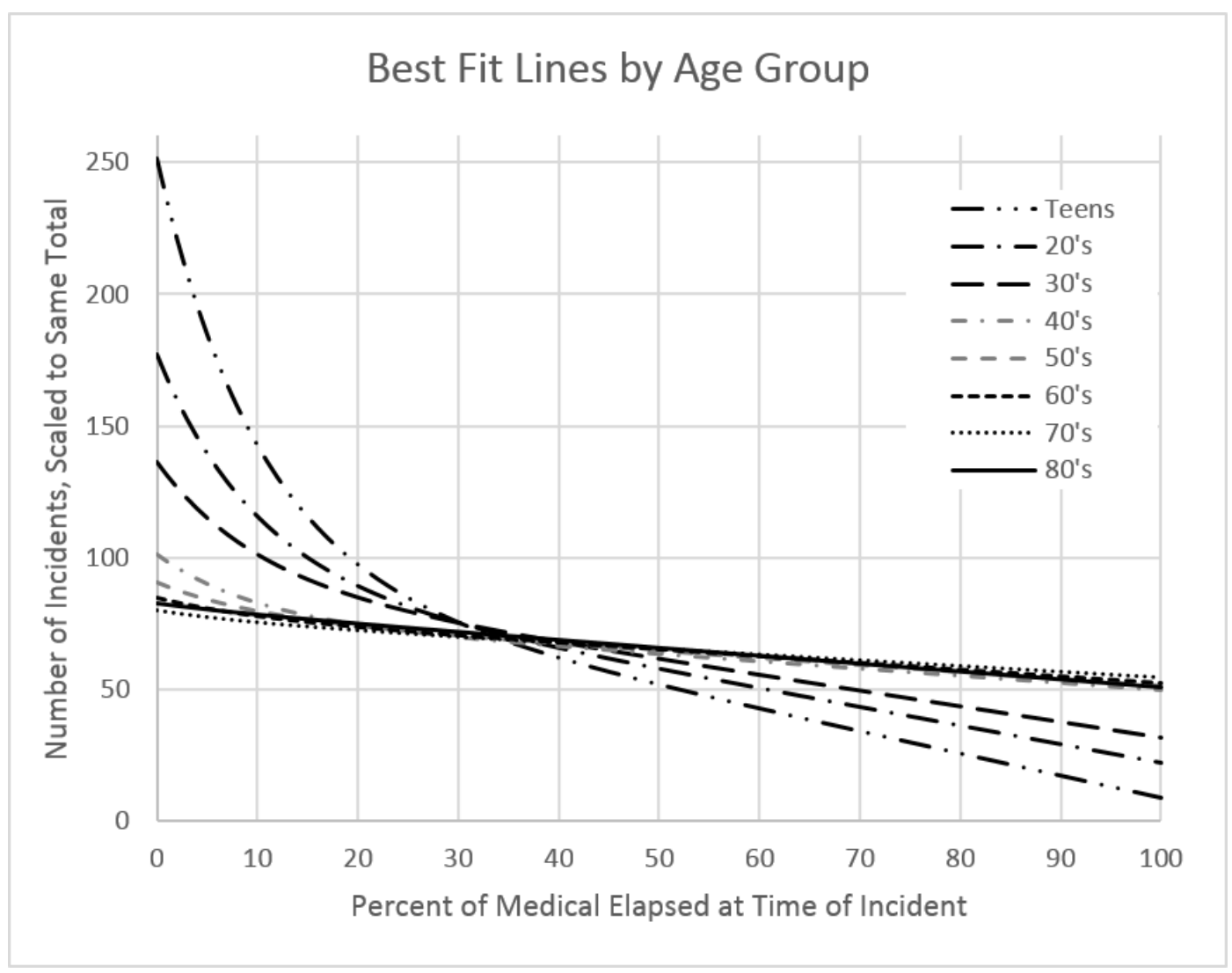

Figure 11: Data Across Demographics

In all age demographics, the exponential portion of the data faded into a relatively linear region with a much lower slope. Here, also, an interesting trend was observed. Increasing age corresponds to slopes that are closer to zero. In other words, the older a pilot is, the less strong the correlation between recent $3^{\text {rd }}$ class medical certification is to likelihood of being involved in an incident. Table 1 summarizes the slopes of these lines. Note that until approximately 80 years of age, the value of this slope appears to converge to around the value of -0.22 seen in pilots in their 70's. This convergence is consistent with a pilot population that flies increasingly uniform numbers of hours across time. The increase in slope to -0.30 seen in octogenarian pilots is probably not significant, due to the much lower $\mathrm{N}$ and higher values of $\mathrm{S}$ seen in this population demographic. Another trend that is notable but easily explained is the relatively abrupt change seen in the slopes for those below 40 and those above 40 . This jump is almost certainly tied to the regulatory change in the duration of $3^{\text {rd }}$ class medicals that occurs at 40 years of age. 
Table 1

Line slopes

\begin{tabular}{|l|l|}
\hline \multicolumn{2}{|l|}{$\begin{array}{l}\text { Comparative Slope } \\
\text { by Demographic }\end{array}$} \\
\hline teens & -0.84 \\
20's & -0.71 \\
30's & -0.59 \\
\hline 40 's & -0.27 \\
50's & -0.26 \\
60's & -0.25 \\
70's & -0.22 \\
\hline $80+$ & -0.30 \\
\hline
\end{tabular}

This study verified the relationship between recent $3^{\text {rd }}$ class medical certification and increased likelihood of being involved in incidents or accident reportable to the NTSB, and it did so for all age demographics. It also identified two separate trends that apply consistently across age brackets. The first trend is that younger pilots show a marked increase in initial risk represented by the exponential component of the data fit. This tendency diminishes rapidly with age. The second trend is that as pilots age, the relationship between recent medical certification and increased risk represented by the linear portion of the graph is also reduced in strength.

\section{Future Work}

This study is based on a series of reasonable assumptions related to the types of data fits that should be attempted. The resulting analysis provided no surprising results. Should the assumptions made in this study be demonstrable, much would be achieved in describing the average flight hour characteristics of private pilots across time. To this end, future study in this regard should probably be directed toward mass logbook based data collection of a random sampling of Private Pilots across time to determine if the observed number of hours actually flown agrees with the data and assumptions presented in this study.

\section{About the Author}

Donald R. Morris, Southern Illinois University, is an Assistant Professor of Aviation Technologies. While he teaches Aviation Maintenance, he is a CFI and avid flight enthusiast. 


\section{References}

Bazargan, M. \& Guzhva, V.S. (2007). Factors contributing to fatalities in general aviation. World Review of Intermodal Transportation Research, 1(2), 170-181

Hunter, David R. (1995). Airman research questionnaire: Methodology and overall results (Federal Aviation Administration Report DOT/FAA/AM-95/27). Retrieved from http://www.faa.gov/go/oamtechreports/1990s/1995

Ison, David C. (2015). Comparative Analysis of Accident and Non-Accident Pilots. Journal of Aviation Technology and Engineering. Vol. 4: Iss. 2, Article 2.

Knecht, William R. (2015). Predicting Accident Rates From General Aviation Pilot Total Flight Hours. (Federal Aviation Administration Report DOT/FAA/AM-15/3). Retrieved from http://www.faa.gov/go/oamtechreports/2010s/2015

Morris, Don (2016). Do NTSB Statistics Support Current FAA Third Class Medical Policy? Collegiate Aviation Review International. Vol. 34: Iss. 1, Article 3.

O'Hare, David \& Chalmers, David (1999). The Incidence of Incidents: A Nationwide Study of Flight Experience and Exposure to Accidents and Incidents. The International Journal of Aviation Psychology Vol. 9 , Iss. 1,1999 